OPEN ACCESS

Edited by:

Maria Tereza Dos Santos Correia, Federal University of Pernambuco,

Brazil

Reviewed by:

Catherine Neuwirth,

Hôpital Universitaire de Dijon, France

Ayush Kumar

University of Manitoba, Canada

*Correspondence:

Yuji Morita

yujmor@dpc.agu.ac.jp

Specialty section:

This article was submitted to Antimicrobials Resistance and

Chemotherapy,

a section of the journa

Frontiers in Microbiology

Received: 20 August 2015 Accepted: 22 July 2016 Published: 05 August 2016

Citation:

Morita Y, Nakashima K-i, Nishino K, Kotani $K$, Tomida J, Inoue $M$ and Kawamura Y (2016) Berberine Is a Novel Type Efflux Inhibitor Which Attenuates the MexXY-Mediated Aminoglycoside Resistance in

Pseudomonas aeruginosa.

Front. Microbiol. 7:1223.

doi: 10.3389/fmicb.2016.01223

\section{Berberine Is a Novel Type Efflux Inhibitor Which Attenuates the MexXY-Mediated Aminoglycoside Resistance in Pseudomonas aeruginosa} Yuji Morita ${ }^{1 *}$, Ken-ichi Nakashima ${ }^{2}$, Kunihiko Nishino $^{3}$, Kenta Kotani $^{1}$, Junko Tomida $^{1}$,
Makoto Inoue ${ }^{2}$ and Yoshiaki Kawamura ${ }^{1}$

\footnotetext{
${ }^{1}$ Department of Microbiology, School of Pharmacy, Aichi Gakuin University, Nagoya, Japan, ${ }^{2}$ Laboratory of Medicinal Resources, School of Pharmacy, Aichi Gakuin University, Nagoya, Japan, ${ }^{3}$ Department of Biomolecular Science and Regulation, Institute of Scientific and Industrial Research, Osaka University, Osaka, Japan
}

The emergence and spread of multidrug-resistant $P$. aeruginosa infections is of great concern, as very few agents are effective against strains of this species. Methanolic extracts from the Coptidis Rhizoma (the rhizomes of Coptis japonica var. major Satake) or Phellodendri Cortex (the bark of Phellodendron chinense Schneider) markedly reduced resistance to anti-pseudomonal aminoglycosides (e.g., amikacin) in multidrug-resistant $P$. aeruginosa strains. Berberine, the most abundant benzylisoquinoline alkaloid in the two extracts, reduced aminoglycoside resistance of $P$. aeruginosa via a mechanism that required the MexXY multidrug efflux system; berberine also reduced aminoglycoside MICs in Achromobacter xylosoxidans and Burkholderia cepacia, two species that harbor intrinsic multidrug efflux systems very similar to the MexXY. Furthermore this compound inhibited MexXY-dependent antibiotic resistance of other classes including cephalosporins (cefepime), macrolides (erythromycin), and lincosamides (lincomycin) demonstrated using a pseudomonad lacking the four other major Mex pumps. Although phenylalanine-arginine beta-naphthylamide $(\mathrm{PA} \beta \mathrm{N})$, a well-known efflux inhibitor, antagonized aminoglycoside in a MexXY-dependent manner, a lower concentration of berberine was sufficient to reduce amikacin resistance of $P$. aeruginosa in the presence of PABN. Moreover, berberine enhanced the synergistic effects of amikacin and piperacillin (and vice versa) in multidrug-resistant $P$. aeruginosa strains. Thus, berberine appears to be a novel type inhibitor of the MexXY-dependent aminoglycoside efflux in P. aeruginosa. As aminoglycosides are molecules of choice to treat severe infections the clinical impact is potentially important.

Keywords: Pseudomonas aeruginosa, efflux, mexXY, aminoglycoside resistance, berberine 


\section{INTRODUCTION}

Pseudomonas aeruginosa is a metabolically versatile bacterium that can cause a wide range of severe opportunistic infections in patients with serious underlying medical conditions (Gellatly and Hancock, 2013). Infections caused by $P$. aeruginosa often are hard to treat; inappropriate chemotherapy readily selects multidrugresistant $P$. aeruginosa against which very few agents are effective (Poole, 2011; Morita et al., 2014). This so-called "antibiotic resistance crisis" has been compounded by the lag in antibiotic discovery and development programs in recent years, and is jeopardizing the essential role played by antibiotics in current medical practices (Rossolini et al., 2014). Moreover, P. aeruginosa possesses an intrinsic resistance to many antimicrobials because of the bacterium's outer-membrane barrier, the presence of multidrug efflux transporters, and endogenous antimicrobial inactivation (Poole, 2011; Morita et al., 2015b). Although, antipseudomonal agents (e.g., carbapenems) have been discovered and developed, $P$. aeruginosa readily acquires resistance to individual agents via chromosomal mutations and lateral gene transfer (Poole, 2011; Morita et al., 2015b).

The resistance-nodulation-division (RND) efflux pumps play a major role in multidrug resistant phenotype attributed to both acquired and intrinsic mechanisms of resistance in $P$. aeruginosa (Poole, 2011, 2013). This pathogen expresses several threecomponent RND-type multidrug efflux systems, among which four, MexAB-OprM, MexCD-OprJ, MexEF-OprN, and MexXYOprM (OprA), are reported to be significant determinants of multidrug resistance in lab and clinical isolates (Poole, 2013; Li et al., 2015). Unlike MexAB-OprM and MexXY-OprM, which contribute to intrinsic resistance, the MexEF-OprN and MexCDOprJ systems are typically quiescent in wild-type cells (Poole, 2013). The tripartite structure consists of an integral membrane efflux transporter with broad substrate specificity (MexB, MexD, MexF, MexY), an outer membrane channel (OprM, OprJ, OprN, OprA), and a periplasmic protein adapter (MexA, MexC, MexE, MexX) (Li et al., 2015). The RND pumps use the proton motive force to capture antimicrobials from the periplasmic space and directly to extrude antimicrobials out of the cell (Li et al., 2015). Among them, the MexXY system is the only significant determinant of efflux-mediated aminoglycoside resistance in P. aeruginosa (Morita et al., 2012a).

The phenyl-arginine- $\beta$-naphthylamide (PA $\beta N$, MC-207,110) is well-known as a non-specific inhibitor against the RND-type multidrug efflux pumps in $P$. aeruginosa (Lomovskaya et al., 2001). However it antagonized the anti-pseudomonas activity of aminoglycosides (amikacin and netilmicin) in a MexXYdependent manner even though it inhibited MexXY-dependent fluoroquinolone (levofloxacin) resistance of P. aeruginosa (Mao et al., 2001). MP 601384, the only inhibitor of the MexXYmediated aminoglycoside resistance has been reported to date (Jassem et al., 2011) although its chemical structure was not revealed.

In the current study we identify berberine, a natural isoquinoline alkaloid produced by a variety of plant species (Tillhon et al., 2012), potentiates aminoglycoside activity against $P$. aeruginosa including multidrug resistant isolates through screening from traditional Japanese herbal preparations, frequently prescribed as Kampo prescriptions (Watanabe et al., 2011). This compound has been reported as a potent efflux inhibitor against Gram-positive bacteria such as Staphylococcus aureus (Tillhon et al., 2012) but it has a weak activity against Gram-negative bacteria such as $P$. aeruginosa in part due to a substrate of multidrug efflux pumps (Tegos et al., 2002).

\section{MATERIALS AND METHODS}

\section{Bacterial Strains, Plasmids, and Growth Conditions}

The bacterial strains and plasmids used in this study are listed in Table 1. Of note various $P$. aeruginosa strains in which contribution of the MexXY efflux system to aminoglycoside resistance were assessed before (Morita et al., 2012a) are used in the main (PAO1; a reference strain, PAGU 1498 and PAGU 1606; multidrug resistant clinical isolates, PAGU 1569; panaminoglycoside clinical isolates) (Table 1). Among the four strains PAGU 1498 is the agrZ-type MexXY-overproducing mutant (Morita et al., 2012a).

Bacterial cells were grown (unless otherwise indicated) in Luria (L) broth and on L agar (1.5\%) under aerobic conditions at $37^{\circ} \mathrm{C}$, as previously described, with antibiotics as specified (Morita et al., 2015b). Bacterial growth was quantified by measuring the optical density at $600 \mathrm{~nm}$ on an Ultrospec 2100 Pro Spectrophotometer (GE Healthcare Corp., Tokyo, Japan), unless otherwise indicated. Cells harboring the plasmid pEX18Tc (Hoang et al., 1998) or derivatives thereof were maintained on medium supplemented with $2.5-10 \mu \mathrm{g} / \mathrm{ml}$ tetracycline for E. coli or and selected on medium supplemented with $20-150 \mu \mathrm{g} / \mathrm{ml}$ tetracycline for P. aeruginosa. Cells harboring the plasmid pFLP2 (Hoang et al., 1998) were maintained and selected on medium supplemented with $100 \mu \mathrm{g} / \mathrm{ml}$ ampicillin for E. coli or 50-200 $\mu \mathrm{g} / \mathrm{ml}$ carbenicillin for $P$. aeruginosa.

\section{Construction of $P$. aeruginosa Mutants}

In-frame deletion mutants and/or aacC1-gfp insertion mutants of mexXY, mex $Z$, and mex $V W$ from $P$. aeruginosa PAO1 derivatives were constructed using the previously described $s a c B$-based strategy (Morita et al., 2006, 2010, 2015a). The plasmids and resulting $P$. aeruginosa mutants are listed in Table $\mathbf{1}$, while the primer pairs are listed in Table 2. To introduce gene deletions into strains of $P$. aeruginosa, deletion constructs were first prepared in plasmid pEX18Tc by cloning PCR-amplified 0.75$\mathrm{kb}$ DNA fragments corresponding to the regions upstream and downstream of the gene sequences to be deleted. The selection concentrations of tetracycline and gentamicin for the recombination events were adjusted to reflect the endogenous tetracycline and gentamicin MICs of the P. aeruginosa strains. These constructs were confirmed by colony PCR.

\section{Preparation of Crude Drug Extracts}

A total of 96 crude drugs (listed in Table S1) that are used in Kampo prescriptions in Japan (Tanabe et al., 2014) were prepared as follows. Ten grams of each crude drug was placed into 100 $\mathrm{ml}$ of methanol and extracted for 1 day at room temperature, 
TABLE 1 | Bacterial strains and plasmids.

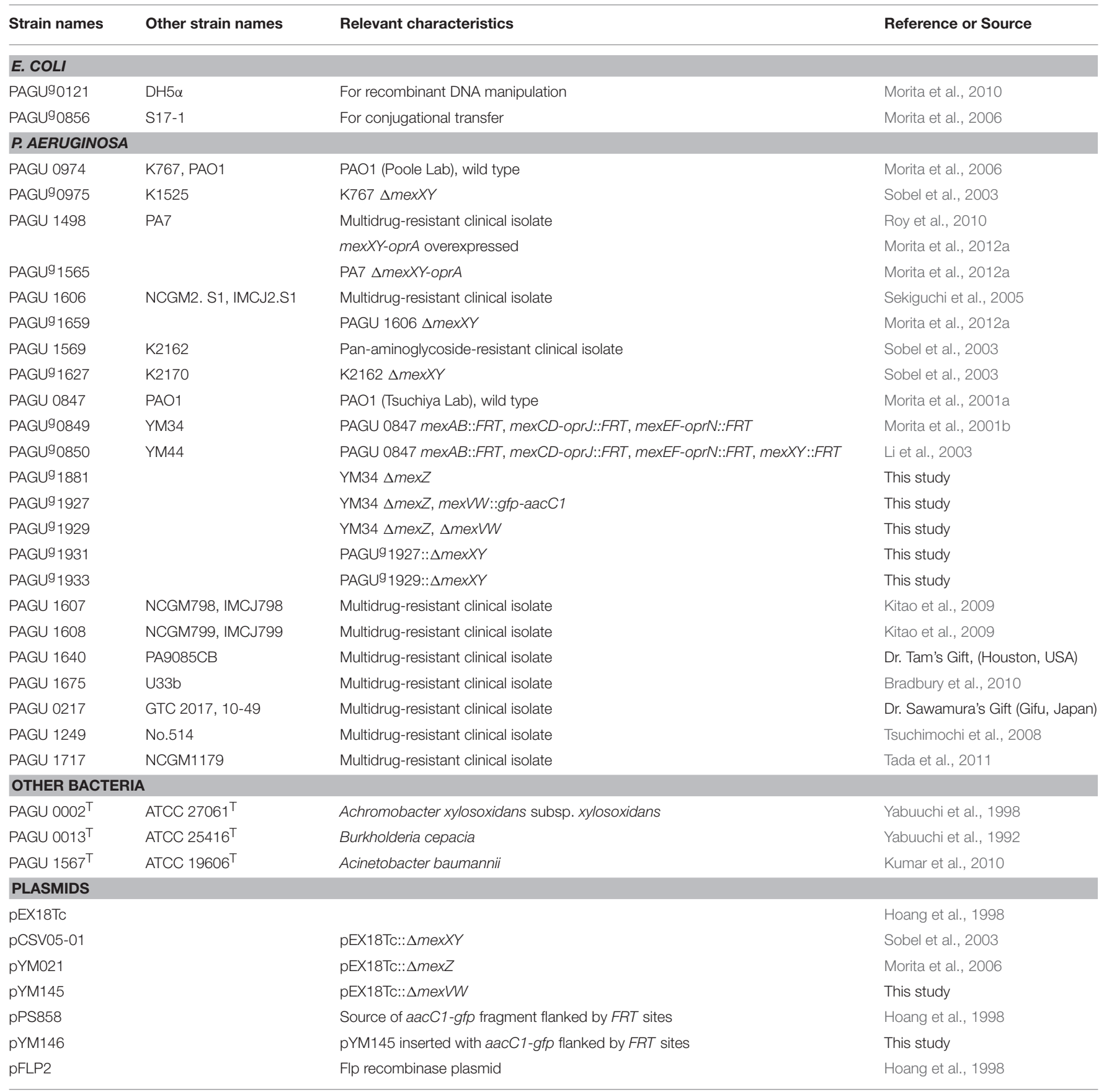

TABLE 2 | Primers used in this study.

\begin{tabular}{llll}
\hline Primer & Sequence $\left(\mathbf{5}^{\prime} \mathbf{-} \mathbf{3}^{\prime}\right)$ & Purpose & References \\
\hline Sacl-mexWW-UF & GCTAGAGCTCCTGGTAGTGGCCAACGGCG & mexWW genes disruption of $P$. aeruginosa PAO1 derivatives & This study \\
BamHI-mexWW-UR & CTGAGGATCCCATAATCCTGGTCCCTGGTATGCC & mexWW genes disruption of $P$. aeruginosa PAO1 derivatives & This study \\
BamHI-mexWW-DF & GCATGGATCCTGATCGGAAACGGCGGAC & mexWW genes disruption of $P$. aeruginosa PAO1 derivatives & This study \\
Hindlll-mexWW-DR & CTAGAAGCTITCCGAGGGGCTTGAGGT & mexWW genes disruption of $P$. aeruginosa PAO1 derivatives & This study
\end{tabular}


and then the solution was filtered. The extraction process was performed three times. Each filtrate was mixed and concentrated in vacuo at $40^{\circ} \mathrm{C}$ by using a rotary evaporator N-1000 (EYELA, Tokyo, Japan) equipped with a coolant system CCA-1110 (EYELA, Tokyo, Japan). Each final extract was dissolved in dimethyl sulfoxide (DMSO) and adjusted to the concentration of $100 \mathrm{mg} / \mathrm{ml}$. Each of the crude drugs was resuspended at $1 \mathrm{mg} / \mathrm{ml}$ in DMSO, and evaluated on the condition of the broth microdilution method described above at the Section of Preparation of Crude Drug Extracts for the ability to restore effectiveness of either $16 \mu \mathrm{g} / \mathrm{ml}$ imipenem, $4 \mu \mathrm{g} / \mathrm{ml}$ ciprofloxacin, or $32 \mu \mathrm{g} / \mathrm{ml}$ amikacin against highly multidrug-resistant $P$. aeruginosa PAGU 1606.

\section{Antibiotic Susceptibility Assay}

The susceptibility of $P$. aeruginosa to antimicrobial agents in cation-adjusted Mueller-Hinton broth was assessed using the two-fold serial micro-titer broth dilution method described previously (Morita et al., 2012a). Minimal inhibitory concentrations (MICs) were defined as the lowest concentration of antibiotic resulting in visible inhibition of growth after about $18-22 \mathrm{~h}$ of incubation at $37^{\circ} \mathrm{C}$ (for $P$. aeruginosa) or after about 20-24 h of incubation at $35^{\circ} \mathrm{C}$ (for Achromobacter xylosoxidans, Burkholderia cepacia, and Acinetobacter baumannii). The categorization as susceptible, intermediate, and resistant was performed according to the interpretive standards of the Clinical and Laboratory Standards Institute (CLSI).

The fractional inhibitory concentration (FIC) index was calculated as reported elsewhere (Lomovskaya et al., 2001). The effects of the drugs were interpreted to be indicative of synergy when the index was $\leq 0.5$.

Amikacin, ampicillin, azithromycin, berberine, carbenicillin, ciprofloxacin, erythromycin, gentamicin, lincomycin, and tetracycline were purchased from Wako Pure Chemicals Industries, Ltd. (Osaka, Japan). Phenylalanine-arginine $\beta$ naphthylamide was purchased from Sigma-Aldrich Co. LLC (Tokyo, Japan). Imipenem/cilastatin and cefepime were purchased from Sandoz K.K. (Tokyo, Japan). Piperacillin was purchased from Nichi-Iko Pharmaceutical Co., Ltd. (Toyama, Japan). Tobramycin was purchased from Towa Pharmaceutical Co., Ltd. (Kadama, Osaka, Japan). Arbekacin was purchased from Shiono Chemical Co., Ltd. (Fujioka, Gunma, Japan).

\section{High Performance Liquid Chromatographic Method for Quantification of Berberine and Coptisine}

The stock solutions of berberine and coptisine were both prepared in methanol. The extracts also were dissolved in methanol to a concentration of $1 \mathrm{mg} / \mathrm{ml}$. In order to construct calibration curves, a series of standard solution of each compound was prepared by appropriate dilution of the stock solutions. Calibration curves were constructed by plotting the peak area ratio vs. the concentration.

The high performance liquid chromatographic conditions were as follows: pump, Hitachi L-2130; autosampler, Hitachi L2200; detector, Hitachi L-2455; column, YMC Pack ODS-AP302
(4.6 x $150 \mathrm{~mm}$, YMC); mobile phase, $0.1 \mathrm{M}$ phosphate buffer ( $\mathrm{pH}$ 2.1)-methanol (70:30, v/v); flow rate, $1 \mathrm{ml} / \mathrm{min}$; column temperature, $25^{\circ} \mathrm{C}$; DAD, $340 \mathrm{~nm}$; and injection volume, $10 \mu \mathrm{l}$.

\section{Molecular Biology Techniques}

Plasmid DNA isolation from E. coli, DNA purification, measurement of DNA concentration, DNA digestion with restriction enzymes, DNA dephosphorylation, DNA ligation, isolation of chromosomal DNA from P. aeruginosa, PCR conditions, nucleotide sequencing, competent cell preparation from E. coli, transformation of E. coli, and transfer of plasmids into $P$. aeruginosa via conjugation were performed as described previously (Morita et al., 2015b), unless otherwise indicated. DNA sequences and amino acid sequences were analyzed using the Pseudomonas Genome Database (Winsor et al., 2011), Basic Local Alignment Search Tool (BLAST), and the software DNASIS Pro (Ver. 2.1; Hitachi, Japan).

\section{RESULTS}

\section{Isolation of a Novel Inhibitor of the MexXY Multidrug Efflux System from the Rhizomes of Coptis Japonica and the Bark of Phellodendron Amurense}

Extracts from the Coptidis Rhizoma (the rhizomes of Coptis japonica var. major Satake) or Phellodendri Cortex (the bark of Phellodendron chinense Schneider) restored the effectiveness of $32 \mu \mathrm{g} / \mathrm{ml}$ amikacin against the highly multidrug-resistant $P$. aeruginosa PAGU 1606. None of the tested extracts restored susceptibility of the strain to $16 \mu \mathrm{g} / \mathrm{ml}$ imipenem or $4 \mu \mathrm{g} / \mathrm{ml}$ ciprofloxacin (data not shown). The extracts of both Coptis rhizome and Phellodendron bark reduced MICs of amikacin and gentamicin (but not those of imipenem or ciprofloxacin) by eight-fold or more in the two multidrug resistant $P$. aeruginosa strains (PAGU 1606 and PAGU 1498) (Table 3).

\section{Berberine Is a Novel Inhibitor of Aminoglycoside Resistance Dependent on the MexXY-Efflux System in $P$. aeruginosa}

Berberine is reported the most abundant benzylisoquinoline alkaloid in the two active extracts (Tillhon et al., 2012). Therefore, we next tested the activity of berberine along with coptisine, a similar benzylisoquinoline alkaloid. Berberine constituted 34.4\% of the extract from the rhizomes of C. japonica and $28.3 \%$ of extract from the bark of Phellodendron amurense (Figure S1). Coptisine was the second-most abundant benzylisoquinoline alkaloid from the Coptis extract, constituting $4.1 \%$ of the extract from the rhizomes of $C$. japonica (Figure S1). Although berberine was ineffective as a solo antibacterial (with an MIC >512 $\mu \mathrm{g} / \mathrm{ml}$ MIC against $P$. aeruginosa; data not shown), berberine inhibited aminoglycoside resistance by two- to > eight-fold against four $P$. aeruginosa strains (Table 4). Such a reduction was not observed in isogenic mutants deficient in the RND-type multidrug efflux system MexXY, which is considered one of the major aminoglycoside resistance determinants in $P$. aeruginosa (Morita et al., 2012b) (Table 4). Coptisine showed activity similar 
TABLE 3 | Methanol extracts from the Coptidis Rhizoma restore aminoglaycoside susceptibility in multidrug-resistant $P$. aeruginosa.

\begin{tabular}{|c|c|c|c|c|c|c|c|c|c|c|c|}
\hline \multirow[t]{2}{*}{ P. aeruginosa Strain } & \multirow[t]{2}{*}{ Relevant property } & \multicolumn{10}{|c|}{$\operatorname{MIC}(\mu \mathrm{g} / \mathrm{ml})^{*}$} \\
\hline & & - & + & - & + & - & + & - & + & - & + \\
\hline PAGU 1606 & Multidrug resistant & 256 & 32 & 64 & 8 & 64 & 64 & $>128$ & $>128$ & 128 & 128 \\
\hline PAGU 1498 & Multidrug resistant & 32 & 4 & $>512$ & 64 & 128 & 64 & 32 & 16 & 1 & 2 \\
\hline PAO1 (PAGU 0974) & Wild type & 2 & 1 & 2 & 0.5 & 0.25 & 0.25 & 4 & 4 & 0.5 & 1 \\
\hline
\end{tabular}

AMK, amikacin; GEN, gentamicin; CIP, ciprofloxacin; FEP, cefepime; IPM, imipenem.

${ }^{\star}$ MICs are measured in the absence (-) or presence (+) of methanol extract from the Coptidis Rhizoma.

TABLE 4 | Berberine attenuates aminoglycoside resistance of $P$. aeruginosa in the MexXY -dependent manner.

\begin{tabular}{|c|c|c|c|c|c|c|c|c|c|}
\hline \multirow[t]{2}{*}{ Strains } & \multirow[t]{2}{*}{ Relevant property } & \multicolumn{8}{|c|}{ MIC $(\mu \mathrm{g} / \mathrm{ml})$ with $(+)$ and without $(-)$ addition of $256 \mu \mathrm{g} / \mathrm{ml} \mathrm{BB}$} \\
\hline & & - & + & - & + & - & + & - & + \\
\hline PAGU 1606 & Multidrug resistant & 256 & 32 & 32 & 4 & 64 & 16 & 256 & 64 \\
\hline PAGU91659 & PAGU $1606 \triangle X Y$ & 8 & 4 & 1 & 0.5 & 1 & 0.5 & 16 & 8 \\
\hline PAGU 1498 & Multidrug resistant & 32 & 4 & 32 & 4 & $>512$ & 128 & $>256$ & 32 \\
\hline PAGU 1569 & Pan-AG resistant & 256 & 64 & 128 & 16 & 256 & 64 & 32 & 16 \\
\hline PAGU91627 & PAGU $1569 \Delta X Y$ & 32 & 16 & 16 & 16 & 8 & 8 & 4 & 4 \\
\hline PAGU 0974 & Wild type, PAO1 & 2 & 1 & 1 & 0.5 & 2 & 1 & 0.5 & 0.25 \\
\hline PAGU90975 & PAGU $0974 \Delta X Y$ & 0.5 & 0.5 & 0.25 & 0.25 & 0.25 & 0.25 & 0.25 & 0.125 \\
\hline
\end{tabular}

$A G$, aminoglycoside; $A M K$, amikacin; $A B K$, arbekacin; $B B$, berberine; GEN, gentamicin; TOB, tobramycin; $X Y$, mexXY.

to that of berberine (data not shown). These results indicate that the inhibitory activity of the two extracts reflected, at least in part, the activity of berberine and/or coptisine. Of note berberine also inhibited aminoglycoside resistance of $A$. xylosoxidans PAGU $0002^{\mathrm{T}}$ and B. cepacia PAGU $0013^{\mathrm{T}}$ but not that of $A$. baumannii PAGU $1567^{\mathrm{T}}$ (Table 5).

\section{Berberine Inhibits MexXY-Mediated Resistance to Erythromycin, Lincomycin, and Cefpirome in a $P$. aeruginosa Mutant Lacking Four RND Multidrug Efflux Systems (MexAB, MexCD, MexEF, and MexVW)}

To test the effect of berberine on non-aminoglycoside susceptibility, we, first of all, evaluated MICs against the wild-type strain (PAO1) and then, in order to remove any effects of the RND pumps, evaluated MICs in a multidrug-sensitive $P$. aeruginosa strain mutated in the loci encoding four RND multidrug efflux systems (mexXY, mexAB, mexCD-oprJ, and mexEF-oprN) (Morita et al., 2001b; Li et al., 2003) (Table 6). Like the parent strain, the quadruple efflux mutant (PAGU ${ }^{\mathrm{g}}$ 0850) also exhibited an eight-fold decrease in MIC of erythromycin in the presence of berberine (Table 6). However mutation of one of the remaining RND multidrug efflux systems (mexVW) attenuated the effect of berberine, with the quintuple mutant (PAGU $^{g}$
TABLE 5 | Berberine attenuates aminoglycoside resistance of A. xylosoxidans and B. cepacia.

Strains $\quad$ MIC $(\mu \mathrm{g} / \mathrm{ml})$ with $(+)$ and without $(-)$ addition of 256 $\mu \mathrm{g} / \mathrm{ml} \mathrm{BB}$

\begin{tabular}{|c|c|c|c|c|c|c|c|c|}
\hline & \multicolumn{2}{|c|}{ AMK } & \multicolumn{2}{|c|}{ ABK } & \multicolumn{2}{|c|}{ GEN } & \multicolumn{2}{|c|}{ ТОВ } \\
\hline & - & + & - & + & - & + & - & + \\
\hline $\begin{array}{l}\text { A. xylosoxidans } \\
\text { PAGU } 0002^{\top}\end{array}$ & $>512$ & 64 & $>512$ & 64 & $>512$ & 16 & 512 & 16 \\
\hline $\begin{array}{l}\text { B. серасiа } \\
{\text { PAGU } 0013^{\top}}^{\top}\end{array}$ & 256 & 16 & 128 & 16 & 256 & 16 & 256 & 8 \\
\hline $\begin{array}{l}\text { A. baumannii } \\
\text { PAGU } 1567^{\top}\end{array}$ & 32 & 32 & 32 & 32 & 64 & 64 & 8 & 8 \\
\hline
\end{tabular}

AMK, amikacin; ABK, arbekacin; BB, berberine; GEN, gentamicin; TOB, tobramycin.

1933) exhibiting only a two-fold decrease in erythromycin MIC upon exposure to berberine (Table 6). Similar effects were seen for lincomycin resistance (Table 6). These results suggest that berberine attenuation of resistance to the macrolides and lincomycin depends on the MexVW multidrug efflux system. The molecular mechanism underlying the berberine effects on the MexVW system will be the subject of further research.

We sought to better understand the molecular mechanism of berberine attenuation of the MexXY-mediated efflux. Therefore, adjuvant effects of berberine on resistance to various 
TABLE 6 | Berberine inhibits MexXY- or MexVW-mediated resistance of $P$. aeruginosa mutants.

\begin{tabular}{|c|c|c|c|c|c|c|c|c|c|c|c|c|}
\hline \multirow[t]{3}{*}{ Strain } & \multirow[t]{3}{*}{ Relevant property } & \multirow[t]{3}{*}{ mex gene* present } & \multicolumn{10}{|c|}{ MIC $(\mu \mathrm{g} / \mathrm{ml})$ with $(+)$ and without $(-)$ addition of $256 \mu \mathrm{g} / \mathrm{ml}$ BB } \\
\hline & & & \multicolumn{2}{|c|}{ ERY } & \multicolumn{2}{|c|}{ LIN } & \multicolumn{2}{|c|}{ FEP } & \multicolumn{2}{|c|}{ CIP } & \multicolumn{2}{|c|}{ TET } \\
\hline & & & - & + & - & + & - & + & - & + & - & + \\
\hline PAGU 0974 & $\begin{array}{l}\text { Wild type, } \\
\text { PAO1 }\end{array}$ & $\begin{array}{l}A B M \\
C D J \\
E F N \\
X Y \\
W W\end{array}$ & 256 & 64 & $>4096$ & 4096 & nd & nd & 0.25 & 0.25 & 32 & 16 \\
\hline PAGU90850 & $\begin{array}{l}\triangle A B \\
\triangle C D J \\
\triangle E F N \\
\triangle X Y\end{array}$ & $\begin{array}{l}M \\
W W\end{array}$ & 64 & 8 & 1024 & 16 & nd & nd & nd & nd & nd & nd \\
\hline PAGU9 1929 & $\begin{array}{l}\triangle A B \\
\triangle C D J \\
\triangle E F N \\
\triangle W W\end{array}$ & $\begin{array}{l}M \\
X Y\end{array}$ & 256 & 32 & 2048 & 256 & 2 & 0.25 & 0.125 & 0.015 & 4 & 0.5 \\
\hline PAGU91933 & $\begin{array}{l}\triangle A B \\
\triangle C D J \\
\triangle E F N \\
\triangle X Y \\
\triangle W W\end{array}$ & $M$ & 8 & 4 & 16 & 8 & 0.125 & 0.125 & 0.008 & 0.002 & 0.03 & 0.008 \\
\hline
\end{tabular}

*mex genes here mean mexAB-oprM, mexCD-oprJ, mexEF-oprN, mexXY, and mexWW.

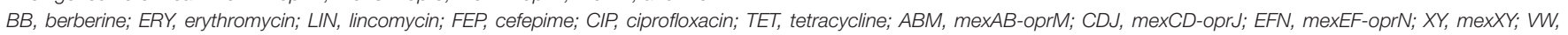
mexVW; $A B$, mexAB; $M$, oprM; nd, not done.

antimicrobial substrates of the MexXY efflux pump were compared in two RND mutant strains differing only by the absence or presence of mexXY (PAGU 1929 vs PAGU ${ }^{g}$ 1933, respectively; Table 6). Specifically, the mexXY genes were overexpressed in PAGU 1933 due to deletion of the mexZ repressor gene. The effects of mexXY overexpression were being determined in the absence of other major RND multidrug efflux systems, since the two strains shared deletions in the mex $A B$, mexCD-oprJ, mexEF-oprN, and mexVW loci. As seen in the earlier experiments (e.g., Table 6), resistance to erythromycin, lincomycin, cefpirome, and aminoglycosides was attenuated eight-fold in the presence of berberine, and these effects were observed only in the presence of the MexXY system (Table 6). While resistance to ciprofloxacin and tetracycline also was attenuated eight-fold in the presence of berberine, a partial berberine effect (four-fold decrease in MIC) was observed for ciprofloxacin and tetracycline even in the absence of mexXY overexpression (compare PAGU 1929 and PAGU ${ }^{g}$ 1933; Table 6). It might suggest that berberine possibly has another target sites other than $5 \mathrm{RND}$ pumps which are involved in resistance to ciprofloxacin and tetracycline. In control experiments (data not shown), we confirmed that berberine did not display antimicrobial activity (at concentrations up to $512 \mu \mathrm{g} / \mathrm{ml}$ ) in the PAGU 1929 strain (that is, despite deletion of mexAB, mexCD-oprJ, mexEF-oprN, and mexVW). We additionally confirmed that the attenuation activity of berberine in the PAGU 1929 background was still concentrationdependent; the aminoglycoside adjuvant activity of berberine in this strain was not detected at a berberine concentration of 8 $\mu \mathrm{g} / \mathrm{ml}$ (data not shown).

\section{Comparison of Berberine with the Known Efflux Inhibitor Phenylalanine-Arginine $\beta$-Naphthylamide (PA $\beta N$ )}

We compared the effects of berberine with those of the previously reported efflux inhibitor $\mathrm{PA} \beta \mathrm{N}$. Consistent with earlier reports (Mao et al., 2001), PA $\beta \mathrm{N}$ did not reduce MexXY-dependent gentamicin resistance (as seen in PAGU ${ }^{g}$ 1929), but PA $\beta N$ did inhibit ciprofloxacin resistance in the quadruple efflux mutant (mexAB, mexCD-oprJ, mexEF-oprN, and mexVW; PAGUg 1929) (Table 7). This distinction suggests that berberine inhibits the MexXY system via a mechanism distinct from that of PA $\beta N$. Given that berberine is known to be substrates of many multidrug efflux pumps, including RND-type multidrug efflux pumps (Tegos et al., 2002), PA $\beta \mathrm{N}$ is expected to facilitate accumulation of berberine in $P$. aeruginosa cells. Indeed, exposure to $\mathrm{PA} \beta \mathrm{N}$ provided inhibition of MexXY-dependent aminoglycoside resistance at a lower concentration of berberine, as shown for the multidrug-resistant $P$. aeruginosa strain PAGU 1606 (Table S2).

\section{Berberine Synergistically Inhibits MexXY-Mediated Aminoglycoside Resistance in $P$. aeruginosa}

FIC indices were determined in multiple strains for the combination of gentamicin and berberine (Table 8). The combination exhibited synergy only in MexXY-positive strains (PAGU' 1927 and PAGU' 1929). This result suggests that berberine and gentamicin act synergistically on the MexXY system. Puzzlingly, berberine MICs in the presence of gentamicin 
are significantly higher in the MexXY-deficient $P$. aeruginosa mutant cells than in the MexXY-overproducing $P$. aeruginosa mutant cells (Table 8). This observation implies that berberine accumulates more effectively in the presence of the MexXY system, although classically the pump facilitates the efflux of substrates.

\section{Berberine Promotes Combined Effects of Amikacin with Piperacillin (and Vice Versa) in Multidrug-Resistant $P$. aeruginosa Strains}

Aminoglycoside uptake is known to be facilitated by inhibitors of bacterial cell wall synthesis such as $\beta$-lactams (Taber et al., 1987). Anti-pseudomonal $\beta$-lactams and polymyxins thus are expected to promote the adjuvant effects of berberine observed above. We tested several such agents (data not shown) and found that piperacillin, an anti-pseudomonal $\beta$-lactam, was among the most potent. The effects of this cell wall inhibitor were seen in all

TABLE 7 | PA $\beta N$ inhibits MexXY-mediated resistance to ciprofloxacin but not to gentamicin, in $P$. aeruginosa mutants.

\begin{tabular}{|c|c|c|c|c|c|c|c|}
\hline \multirow[t]{3}{*}{ Strain } & \multirow[t]{3}{*}{$\begin{array}{l}\text { Relevant } \\
\text { property }\end{array}$} & \multirow[t]{3}{*}{$\begin{array}{l}\text { mex genes* } \\
\text { present }\end{array}$} & \multicolumn{5}{|c|}{$\begin{array}{c}\text { MIC }(\mu \mathrm{g} / \mathrm{ml}) \text { with }(+) \text { and } \\
\text { without }(-) \text { addition of } \\
\text { PA } \beta N^{\star *}\end{array}$} \\
\hline & & & \multicolumn{2}{|c|}{ GEN } & \multicolumn{2}{|c|}{ CIP } & \multirow[t]{2}{*}{ PA $\beta N$} \\
\hline & & & - & + & - & + & \\
\hline PAGU91929 & $\begin{array}{l}\triangle A B \\
\triangle C D J \\
\triangle E F N \\
\triangle W W\end{array}$ & $\begin{array}{l}M \\
X Y\end{array}$ & 2 & 4 & 0.125 & 0.0078 & 512 \\
\hline PAGU91933 & $\begin{array}{l}\triangle A B \\
\triangle C D J \\
\triangle E F N \\
\triangle W W \\
\triangle X Y\end{array}$ & $M$ & 0.25 & 0.25 & 0.0078 & 0.0039 & 32 \\
\hline
\end{tabular}

*mex genes here mean mexAB-oprM, mexCD-oprJ, mexEF-oprN, mexXY, and mexWW. ${ }^{\star *}$ Concentrations of PA $\beta N$ are 1/8-fold MICs for PA $\beta N$ of PAGU 1929 and PAGUg 1933, respectively.

GEN, gentamicin; CIP, ciprofloxacin; $P A \beta N$, phenylalanine-arginine $\beta$-naphthylamide; $A B M$, mexAB-oprM; CDJ, mexCD-oprJ; EFN, mexEF-oprN; $X Y, \operatorname{mexXY;} W W$, mexVW; $A B$, mexAB; $M$, oprM. of the tested multidrug-resistant $P$. aeruginosa strains (Table 9). Notably, exposure to the combination of piperacillin $(32 \mu \mathrm{g} / \mathrm{ml})$ and berberine $(512 \mu \mathrm{g} / \mathrm{ml})$ reduced the amikacin MIC of PAGU 1606 to a level below the amikacin breakpoint of $32 \mu \mathrm{g} / \mathrm{ml}$ (Table 9). In contrast, exposure to piperacillin or berberine alone did not have as large an effect in reducing amikacin resistance. We additionally noted a reduction in the MIC of piperacillin in PAGU 1606 when grown in the presence of both of berberine (512 $\mu \mathrm{g} / \mathrm{ml})$ and sub-breakpoint amikacin $(16 \mu \mathrm{g} / \mathrm{ml})$, with the piperacillin MIC reduced to a level similar to that seen when grown in the presence of $128 \mu \mathrm{g} / \mathrm{ml}$ of amikacin alone (Table 9). Indeed, the FIC index of amikacin in combination with piperacillin of PAGU 1606 was calculated as follows: amikacin MIC in the presence of piperacillin/amikacin MIC) + (piperacillin MIC in the presence of amikacin/piperacillin MIC) $=64 / 256+16 / 256<0.5$. It suggested synergy of these two compounds in PAGU 1606 (Table 9).

\section{DISCUSSION}

The MexXY multidrug efflux system is a significant determinant of resistance to aminoglycosides in $P$. aeruginosa, although aminoglycosides are not standard RND pump substrates (Morita et al., 2012b). The MexXY pump typically is encoded by a two-gene operon and comprises a periplasmic membrane fusion protein (MexX) and an inner-membrane (IM) drug/ $\mathrm{H}^{+}$ antiporter (MexY; the RND component); the pump is functional only when combined with an outer-membrane (OM) channel encoded by a separate locus (e.g., OprM, which is encoded by the third gene of another multidrug efflux operon, mexAB-oprM Morita et al., 2012b). In some $P$. aeruginosa strains, such as the taxonomic outlier strain PA7, the mexXY genes are adjacent to a third gene (oprA in PA7) encoding an outer membrane channel; the resulting three-gene operon is similar to axyXY-oprZ of $A$. xylosoxidans and amrAB-oprA of various Burkholderia species (e.g., B. pseudomallei and B. cepacia complexes) (Morita et al., 2012a,b). In the PA7-related strains, MexXY seem to cooperate with both OprM and OprA, as either of these Opr proteins can compensate for the genetically engineered suppression of the other (Morita et al., 2012b; Li et al., 2015). In P. aeruginosa, the MexXY system mediates resistance to aminoglycosides as well as various clinically relevant antimicrobials such as fluoroquinolones, some $\beta$-lactams (e.g., cefepime), tetracyclines

TABLE 8 | Berberine synergistically inhibits MexXY-mediated gentamicin resistance in $P$. aeruginosa mutants.

\begin{tabular}{|c|c|c|c|c|c|c|c|c|}
\hline \multirow[t]{2}{*}{ Strain } & \multirow[t]{2}{*}{$X Y$} & \multirow[t]{2}{*}{$A A C$} & \multicolumn{2}{|c|}{ MIC $(\mu \mathrm{g} / \mathrm{ml})$ for GEN in the presence of: } & \multicolumn{2}{|c|}{ MIC $(\mu \mathrm{g} / \mathrm{ml})$ for BB in the presence of: } & \multirow[t]{2}{*}{ FIC } & \multirow[t]{2}{*}{ Mode of Interaction } \\
\hline & & & - & $+\mathrm{BB}$ & - & +GEN & & \\
\hline PAGUg 1927 & + & + & 1024 & 256 & $>512$ & 128 & $<0.5$ & Synergy \\
\hline PAGU9 1931 & - & + & 8 & 16 & $>512$ & $>512$ & $>2.0$ & Indifferent \\
\hline PAGU9 1929 & + & - & 8 & 2 & $>512$ & 128 & $<0.5$ & Synergy \\
\hline PAGU9 1933 & - & - & 0.25 & 0.25 & $>512$ & $>512$ & $>1.0$ & Indifferent \\
\hline
\end{tabular}

GEN, gentamicin; $B B$, berberine; $X Y$, mexXY; $A A C$, aacC1*.

*aacC1 is a gentamycin acetyl transferase-encoding gene derived from pPS858 (Hoang et al., 1998). 
TABLE 9 | Berberine enhances synergistic effect of piperacillin and amikacin (and vice versa) in multidrug-resistant $P$. aeruginosa strains.

\begin{tabular}{|c|c|c|c|c|c|c|c|c|}
\hline \multirow[t]{2}{*}{ Strain } & \multicolumn{4}{|c|}{ AMK MIC $(\mu \mathrm{g} / \mathrm{ml})$ in the presence of: } & \multicolumn{4}{|c|}{ PIPC MIC $(\mu \mathrm{g} / \mathrm{ml})$ in the presence of: } \\
\hline & - & BB (512) & PIPC (32) & BB (512) PIPC (32) & - & BB (512) & AMK (128) & BB (512) AMK (16) \\
\hline PAGU 1606 & 256 & 64 & 64 & 16 & 256 & 256 & 16 & 32 \\
\hline PAGU 1607 & 256 & nd & nd & 16 & 256 & nd & nd & 32 \\
\hline PAGU 1608 & 256 & nd & nd & 16 & 256 & nd & nd & 32 \\
\hline PAGU 1640 & 64 & nd & nd & 16 & 1024 & nd & nd & 64 \\
\hline PAGU 1675 & $>1024$ & nd & nd & 64 & 1024 & nd & nd & 256 \\
\hline PAGU 0217 & 256 & nd & nd & 8 & 256 & nd & nd & 32 \\
\hline PAGU 1249 & $>1024$ & nd & nd & 4 & 256 & nd & nd & $<0.5$ \\
\hline PAGU 1717 & 128 & nd & nd & 2 & 128 & nd & nd & 8 \\
\hline
\end{tabular}

AMK, amikacin; BB, berberine, PIPC, piperacillin; nd, not done.

Values in parentheses are concentrations $(\mu \mathrm{g} / \mathrm{ml})$ of indicated drugs.

(including tigecycline), and macrolides such as azithromycin (Morita et al., 2012b). In wild-type cells, the mexXY operon is induced by agents that target ribosomes (Jeannot et al., 2005; Morita et al., 2006); the operon is overexpressed in mutant cells (e.g., Morita et al., 2012b; Guénard et al., 2014). Potent inhibitors of RND-type efflux pumps could be used as adjunctive therapies that would increase the potency of existing antibiotics and decrease the emergence of multidrug resistance in Gramnegative pathogens such as $P$. aeruginosa and Enterobacteriaceae (Gill et al., 2015; Opperman and Nguyen, 2015; Venter et al., 2015). Several potent inhibitors of RND-type efflux pump (e.g., PAßN (Lomovskaya et al., 2001), D13-9001 (Nakashima et al., 2013), and MBX2319 Vargiu et al., 2014) have been reported. However, only a single inhibitor of MexXY (MP 601384) has been reported to date (Jassem et al., 2011); the chemical structure of that agent was not revealed.

In the present study, berberine was shown to synergistically inhibit aminoglycoside resistance in $P$. aeruginosa in a MexXY-dependent manner. We observed a similar effect in $A$. xylosoxidans and B. cepacia, distinct species that possess MexXY orthologues (Morita et al., 2012b). On the other hand, we did not observe berberine attenuation of aminoglycoside resistance in A. baumannii. In Acinetobacter, the AdeAB RND-type pump mediates aminoglycoside resistance (Magnet et al., 2001), and the AdeAB system exhibits more sequence similarity to $P$. aeruginosa MexCD than to $P$. aeruginosa MexXY (Morita et al., 2012b). Furthermore, berberine attenuated MexXY-dependent resistance to aminoglycosides in a $P$. aeruginosa harboring MexXY but lacking another four RND multidrug efflux systems. We observed the same effect in this background for antimicrobials of other classes (including erythromycin, cefepime, and lincomycin) that are considered substrates of the MexXY pump (Morita et al., $2012 b$ ). Berberine is a natural isoquinoline alkaloid produced by a variety of plant species; this compound has been reported to possess a number of biological activities, including antimicrobial effects (Tillhon et al., 2012). Recently, berberine's antibacterial properties were shown to be due primarily to inhibition of the cell division protein FtsZ (Domadia et al., 2008; Boberek et al., 2010). However, this compound's antibacterial activity is not strong against Gram-negative bacteria such as $P$. aeruginosa at least in part because many bacterial multidrug efflux pumps recognize berberine as a substrate (e.g., Morita et al., 1998; Tegos et al., 2002). Berberine is more potent against Gram-positive bacteria such as Mycobacterium tuberculosis and $S$. aureus by inhibiting MF-type multidrug efflux pumps such as NorA (Tegos et al., 2002). The MexXY system confers berberine resistance in Escherichia coli lacking the major RND-type mulidrug efflux system AcrAB (data not shown) as well as confers $\mathrm{PA} \beta \mathrm{N}$ resistance in $P$. aeruginosa lacking the major RND pumps (Table 6), suggesting that each of the two agents acts as a competitive inhibitor of substrate binding and/or extrusion. However each spectrum of antimicrobial potentiation is not the same. Unfortunately, the adjuvant activity of berberine requires (in Pseudomonas) a relatively high $(>100 \mu \mathrm{g} / \mathrm{ml})$ concentration of the compound, consistent with a limited ability to inhibit the MexXY efflux. Clinical development would require optimization of the structure of berberine to provide better synergistic activity with aminoglycosides against $P$. aeruginosa. Recently a region of MexY (the substrate-specificity-determining RND component of this pump) that corresponds to a proximal binding pocket of AcrB was shown to be involved in aminoglycoside recognition and efflux (Lau et al., 2014; Li et al., 2015). This protein domain would be a good starting place for understanding details of aminoglycoside recognition and export by MexY. Although the substrate specificity of E. coli AcrAB-TolC (the best studied RND-type multidrug efflux pump) is extraordinarily broad, the complex does not recognize aminoglycosides as substrates (Li et al., 2015). The design and development of better MexXY inhibitors will require improved understanding of the molecular mechanisms of MexXY-mediated aminoglycoside resistance.

In conclusion berberine is the first efflux inhibitor that restores aminoglycosides activity in multidrug resistant $P$. aeruginosa. As aminoglycosides are molecules of choice to treat severe infections the clinical impact is potentially important.

\section{AUTHOR CONTRIBUTIONS}

YM conceived and designed the experiment. YM, KeN, and KK performed the experiments. YM, KeN, KuN, KK, JT, MI, and YK analyzed the data. YM wrote the paper. 


\section{ACKNOWLEDGMENTS}

We thank Ms. Yuki Kojima, Ms. Akane Kusu, Ms. Shiori Fukaya, and Dr. Hiroki Tanabe (School of Pharmacy, Aichi Gakuin University) for their contributions. We thank Dr. R. Keith Poole (Queen's University, Canada), Dr. Nobuyuki Shimono (Kyushu University, Japan), Dr. Teruo Kirikae (National Center for Global Health and Medicine, Japan), Dr. Ayush Kumar (University of Manitoba, Canada), Dr. Vincent H. Tam (University of Houston College of Pharmacy, USA), and Dr. Richard S. Bradbury (University of Tasmania, Australia) for providing bacterial strains. This work was supported in part by a Grant-in-Aid for Scientific Research (C) (Kakenhi 26460080) from the Japan Society for

\section{REFERENCES}

Boberek, J. M., Stach, J., and Good, L. (2010). Genetic evidence for inhibition of bacterial division protein FtsZ by berberine. PLoS ONE 5:e13745. doi: 10.1371/journal.pone.0013745

Bradbury, R. S., Roddam, L. F., Merritt, A., Reid, D. W., and Champion, A. C. (2010). Virulence gene distribution in clinical, nosocomial and environmental isolates of Pseudomonas aeruginosa. J. Med. Microbiol. 59, 881-890. doi: 10.1099/jmm.0.018283-0

Domadia, P. N., Bhunia, A., Sivaraman, J., Swarup, S., and Dasgupta, D. (2008). Berberine targets assembly of Escherichia coli cell division protein FtsZ. Biochemistry 47, 3225-3234. doi: 10.1021/bi7018546

Gellatly, S. L., and Hancock, R. E. (2013). Pseudomonas aeruginosa: new insights into pathogenesis and host defenses. Pathog. Dis. 67, 159-173. doi: 10.1111/2049-632x.12033

Gill, E. E., Franco, O. L., and Hancock, R. E. (2015). Antibiotic adjuvants: diverse strategies for controlling drug-resistant pathogens. Chem. Biol. Drug Des. 85, 56-78. doi: 10.1111/cbdd.12478

Guénard, S., Muller, C., Monlezun, L., Benas, P., Broutin, I., Jeannot, K., et al. (2014). Multiple mutations lead to MexXY-OprM-dependent aminoglycoside resistance in clinical strains of Pseudomonas aeruginosa. Antimicrob. Agents Chemother. 58, 221-228. doi: 10.1128/aac.01252-13

Hoang, T. T., Karkhoff-Schweizer, R. R., Kutchma, A. J., and Schweizer, H. P. (1998). A broad-host-range Flp-FRT recombination system for site-specific excision of chromosomally-located DNA sequences: application for isolation of unmarked Pseudomonas aeruginosa mutants. Gene 212, 77-86.

Jassem, A. N., Zlosnik, J. E., Henry, D. A., Hancock, R. E., Ernst, R. K., and Speert, D. P. (2011). In vitro susceptibility of Burkholderia vietnamiensis to aminoglycosides. Antimicrob. Agents Chemother. 55, 2256-2264. doi: 10.1128/aac.01434-10

Jeannot, K., Sobel, M. L., El Garch, F., Poole, K., and Plésiat, P. (2005). Induction of the MexXY efflux pump in Pseudomonas aeruginosa is dependent on drugribosome interaction. J. Bacteriol. 187, 5341-5346. doi: 10.1128/jb.187.15.53415346.2005

Kitao, T., Miyoshi-Akiyama, T., and Kirikae, T. (2009). AAC(6')-Iaf, a novel aminoglycoside $6^{\prime}-\mathrm{N}$-acetyltransferase from multidrug-resistant Pseudomonas aeruginosa clinical isolates. Antimicrob. Agents Chemother. 53, 2327-2334. doi: 10.1128/aac.01360-08

Kumar, A., Dalton, C., Cortez-Cordova, J., and Schweizer, H. P. (2010). Mini-Tn7 vectors as genetic tools for single copy gene cloning in Acinetobacter baumannii. J. Microbiol. Methods 82, 296-300. doi: 10.1016/j.mimet.2010.07.002

Lau, C. H., Hughes, D., and Poole, K. (2014). MexY-promoted aminoglycoside resistance in Pseudomonas aeruginosa: involvement of a putative proximal binding pocket in aminoglycoside recognition. MBio 5:e01068. doi: 10.1128/mBio.01068-14

Li, X. Z., Plesiat, P., and Nikaido, H. (2015). The challenge of efflux-mediated antibiotic resistance in Gram-negative bacteria. Clin. Microbiol. Rev. 28, 337-418. doi: 10.1128/cmr.00117-14 the Promotion of Science; a research grant from the Research Foundation for Oriental Medicine; and by a research grant from the Institute of Pharmaceutical Life Sciences, Aichi Gakuin University. This work was also performed in part under the Cooperative Research Program (2013315, 2014357, and 2015431) of "Network Joint Research Center for Materials and Devices," collaborated with Dr. KuN (Osaka University, Japan).

\section{SUPPLEMENTARY MATERIAL}

The Supplementary Material for this article can be found online at: http://journal.frontiersin.org/article/10.3389/fmicb. 2016.01223

Li, Y., Mima, T., Komori, Y., Morita, Y., Kuroda, T., Mizushima, T., et al. (2003). A new member of the tripartite multidrug efflux pumps, MexVWOprM, in Pseudomonas aeruginosa. J. Antimicrob. Chemother. 52, 572-575. doi: $10.1093 / \mathrm{jac} / \mathrm{dkg} 390$

Lomovskaya, O., Warren, M. S., Lee, A., Galazzo, J., Fronko, R., Lee, M., et al. (2001). Identification and characterization of inhibitors of multidrug resistance efflux pumps in Pseudomonas aeruginosa: novel agents for combination therapy. Antimicrob. Agents Chemother. 45, 105-116. doi: 10.1128/aac.45.1.105-116.2001

Magnet, S., Courvalin, P., and Lambert, T. (2001). Resistance-nodulationcell division-type efflux pump involved in aminoglycoside resistance in Acinetobacter baumannii strain BM4454. Antimicrob. Agents Chemother. 45, 3375-3380. doi: 10.1128/aac.45.12.3375-3380.2001

Mao, W., Warren, M. S., Lee, A., Mistry, A., and Lomovskaya, O. (2001). MexXY-OprM efflux pump is required for antagonism of aminoglycosides by divalent cations in Pseudomonas aeruginosa. Antimicrob. Agents Chemother. 45, 2001-2007. doi: 10.1128/aac.45.7.2001-2007.2001

Morita, Y., Kimura, N., Mima, T., Mizushima, T., and Tsuchiya, T. (2001a). Roles of MexXY- and MexAB-multidrug efflux pumps in intrinsic multidrug resistance of Pseudomonas aeruginosa PAO1. J. Gen. Appl. Microbiol. 47, 27-32. doi: 10.2323/jgam.47.27

Morita, Y., Kodama, K., Shiota, S., Mine, T., Kataoka, A., Mizushima, T., et al. (1998). NorM, a putative multidrug efflux protein, of Vibrio parahaemolyticus and its homolog in Escherichia coli. Antimicrob. Agents Chemother. 42, 1778-1782.

Morita, Y., Komori, Y., Mima, T., Kuroda, T., Mizushima, T., and Tsuchiya, T. (2001b). Construction of a series of mutants lacking all of the four major mex operons for multidrug efflux pumps or possessing each one of the operons from Pseudomonas aeruginosa PAO1: MexCD-OprJ is an inducible pump. FEMS Microbiol. Lett. 202, 139-143. doi: 10.1111/j.1574-6968.2001.tb10794.x

Morita, Y., Narita, S., Tomida, J., Tokuda, H., and Kawamura, Y. (2010). Application of an inducible system to engineer unmarked conditional mutants of essential genes of Pseudomonas aeruginosa. J. Microbiol. Methods 82, 205-213. doi: 10.1016/j.mimet.2010.06.001

Morita, Y., Sobel, M. L., and Poole, K. (2006). Antibiotic inducibility of the MexXY multidrug efflux system of Pseudomonas aeruginosa: involvement of the antibiotic-inducible PA5471 gene product. J. Bacteriol. 188, 1847-1855. doi: 10.1128/jb.188.5.1847-1855.2006

Morita, Y., Tomida, J., and Kawamura, Y. (2012a). Primary mechanisms mediating aminoglycoside resistance in the multidrug-resistant Pseudomonas aeruginosa clinical isolate PA7. Microbiology 158, 1071-1083. doi: 10.1099/mic.0.054320-0

Morita, Y., Tomida, J., and Kawamura, Y. (2012b). MexXY multidrug efflux system of Pseudomonas aeruginosa. Front. Microbiol. 3:408. doi: 10.3389/fmicb.2012.00408

Morita, Y., Tomida, J., and Kawamura, Y. (2014). Responses of Pseudomonas aeruginosa to antimicrobials. Front. Microbiol. 4:422. doi: 10.3389/fmicb.2013.00422

Morita, Y., Tomida, J., and Kawamura, Y. (2015a). Efflux-mediated fluoroquinolone resistance in the multidrug-resistant Pseudomonas aeruginosa 
clinical isolate PA7: identification of a novel MexS variant involved in upregulation of the mexEF-oprN multidrug efflux operon. Front. Microbiol. 6:8. doi: $10.3389 /$ fmicb. 2015.00008

Morita, Y., Tomida, J., and Kawamura, Y. (2015b). "Resistance and response to anti-pseudomonas agents and biocides," in Pseudomonas: New Aspects of Pseudomonas Biology, eds J. Ramos, J. B. Goldberg, and A. Filloux (New York, NY: Springer), 173-187.

Nakashima, R., Sakurai, K., Yamasaki, S., Hayashi, K., Nagata, C., Hoshino, K., et al. (2013). Structural basis for the inhibition of bacterial multidrug exporters. Nature 500, 102-106. doi: 10.1038/nature12300

Opperman, T. J., and Nguyen, S. T. (2015). Recent advances toward a molecular mechanism of efflux pump inhibition. Front. Microbiol. 6:421. doi: 10.3389/fmicb.2015.00421

Poole, K. (2011). Pseudomonas aeruginosa: resistance to the max. Front. Microbiol. 2:65. doi: 10.3389/fmicb.2011.00065

Poole, K. (2013). "Pseudomonas aeruginosa efflux pumps," in Microbial Efflux Pumps: Current Research, eds E. W. Yu, Q. Zhang, and M. H. Brown (Norfolk: Caiser Academic Press), 175-206.

Rossolini, G. M., Arena, F., Pecile, P., and Pollini, S. (2014). Update on the antibiotic resistance crisis. Curr. Opin. Pharmacol. 18c, 56-60. doi: 10.1016/j.coph.2014.09.006

Roy, P. H., Tetu, S. G., Larouche, A., Elbourne, L., Tremblay, S., Ren, Q., et al. (2010). Complete genome sequence of the multiresistant taxonomic outlier Pseudomonas aeruginosa PA7. PLoS ONE 5:e8842. doi: 10.1371/journal.pone.0008842

Sekiguchi, J., Asagi, T., Miyoshi-Akiyama, T., Fujino, T., Kobayashi, I., Morita, K., et al. (2005). Multidrug-resistant Pseudomonas aeruginosa strain that caused an outbreak in a neurosurgery ward and its aac $\left(6^{\prime}\right)$-Iae gene cassette encoding a novel aminoglycoside acetyltransferase. Antimicrob. Agents Chemother. 49, 3734-3742. doi: 10.1128/aac.49.9.3734-3742.2005

Sobel, M. L., Mckay, G. A., and Poole, K. (2003). Contribution of the MexXY multidrug transporter to aminoglycoside resistance in Pseudomonas aeruginosa clinical isolates. Antimicrob. Agents Chemother. 47, 3202-3207. doi: 10.1128/ AAC.47.10.3202-3207.2003

Taber, H. W., Mueller, J. P., Miller, P. F., and Arrow, A. S. (1987). Bacterial uptake of aminoglycoside antibiotics. Microbiol. Rev. 51, 439-457.

Tada, T., Kitao, T., Miyoshi-Akiyama, T., and Kirikae, T. (2011). Genome sequence of multidrug-resistant Pseudomonas aeruginosa NCGM1179. J. Bacteriol. 193, 6397. doi: 10.1128/jb.06129-11

Tanabe, H., Yasui, T., Kotani, H., Nagatsu, A., Makishima, M., Amagaya, S., et al. (2014). Retinoic acid receptor agonist activity of naturally occurring diterpenes. Bioorg. Med. Chem. 22, 3204-3212. doi: 10.1016/j.bmc.2014.03.047

Tegos, G., Stermitz, F. R., Lomovskaya, O., and Lewis, K. (2002). Multidrug pump inhibitors uncover remarkable activity of plant antimicrobials. Antimicrob. Agents Chemother. 46, 3133-3141. doi: 10.1128/AAC.46.10.31333141.2002
Tillhon, M., Guáman Ortiz, L. M., Lombardi, P., and Scovassi, A. I. (2012). Berberine: new perspectives for old remedies. Biochem. Pharmacol. 84, 1260-1267. doi: 10.1016/j.bcp.2012.07.018

Tsuchimochi, N., Takuma, T., Shimono, N., Nagasaki, Y., Uchida, Y., and Harada, M. (2008). Antimicrobial susceptibility and molecular epidemiological analysis of clinical strains of Pseudomonas aeruginosa. J. Infect. Chemother. 14, 99-104. doi: 10.1007/s10156-007-0578-8

Vargiu, A. V., Ruggerone, P., Opperman, T. J., Nguyen, S. T., and Nikaido, H. (2014). Molecular mechanism of MBX2319 inhibition of Escherichia coli AcrB multidrug efflux pump and comparison with other inhibitors. Antimicrob. Agents Chemother. 58, 6224-6234. doi: 10.1128/aac.03283-14

Venter, H., Mowla, R., Ohene-Agyei, T., and Ma, S. (2015). RND-type drug e ffl ux pumps from Gram-negative bacteria: molecular mechanism and inhibition. Front. Microbiol. 6:377. doi: 10.3389/fmicb.2015.00377

Watanabe, K., Matsuura, K., Gao, P., Hottenbacher, L., Tokunaga, H., Nishimura, K., et al. (2011). Traditional Japanese Kampo Medicine: Clinical Research between Modernity and Traditional Medicine-The State of Research and Methodological Suggestions for the Future. Evid. Based Complement. Alternat. Med. 2011, 513842. doi: 10.1093/ecam/neq067

Winsor, G. L., Lam, D. K., Fleming, L., Lo, R., Whiteside, M. D., Yu, N. Y., et al. (2011). Pseudomonas Genome Database: improved comparative analysis and population genomics capability for Pseudomonas genomes. Nucleic Acids Res. 39, D596-D600. doi: 10.1093/nar/gkq869

Yabuuchi, E., Kawamura, Y., Kosako, Y., and Ezaki, T. (1998). Emendation of genus Achromobacter and Achromobacter xylosoxidans (Yabuuchi and Yano) and proposal of Achromobacter ruhlandii (Packer and Vishniac) comb. nov., Achromobacter piechaudii (Kiredjian et al.) comb. nov., and Achromobacter xylosoxidans subsp. denitrificans (Ruger and Tan) comb. nov. Microbiol. Immunol. 42, 429-438.

Yabuuchi, E., Kosako, Y., Oyaizu, H., Yano, I., Hotta, H., Hashimoto, Y., et al. (1992). Proposal of Burkholderia gen. nov. and transfer of seven species of the genus Pseudomonas homology group II to the new genus, with the type species Burkholderia cepacia (Palleroni and Holmes 1981) comb. nov. Microbiol. Immunol. 36, 1251-1275.

Conflict of Interest Statement: The authors declare that the research was conducted in the absence of any commercial or financial relationships that could be construed as a potential conflict of interest.

Copyright (c) 2016 Morita, Nakashima, Nishino, Kotani, Tomida, Inoue and Kawamura. This is an open-access article distributed under the terms of the Creative Commons Attribution License (CC BY). The use, distribution or reproduction in other forums is permitted, provided the original author(s) or licensor are credited and that the original publication in this journal is cited, in accordance with accepted academic practice. No use, distribution or reproduction is permitted which does not comply with these terms. 